\title{
Comparative Study of Two Laparoscopic Instrument Tracker Designs for Motion Analysis and Image-Guided Surgery: A Technical Evaluation
}

\author{
J.A. Sánchez-Margallo F.M. Sánchez-Margallo I. Oropesa M. Lucas J. Moreno \\ and E.J. Gómez
}

\begin{abstract}
Laparoscopic instrument tracking systems are a key element in image-guided interventions, which requires high accuracy to be used in a real surgical scenario. In addition, these systems are a suitable option for objective assessment of laparoscopic technical skills based on instrument motion analysis. This study presents a new approach that improves the accuracy of a previously presented system, which applies an optical pose tracking system to laparoscopic practice. A design enhancement of the artificial markers placed on the laparoscopic instrument as well as an improvement of the calibration process are presented as a means to achieve more accurate results. A technical evaluation has been performed in order to compare the accuracy between the previous design and the new approach. Results show a remarkable improvement in the fluctuation error throughout the measurement platform. Moreover, the accumulated distance error and the inclination error have been improved. The tilt range covered by the system is the same for both approaches, from $90^{\circ}$ to $7.5^{\circ}$. The relative position error is better for the new approach mainly at close distances to the camera system.
\end{abstract}

Keywords-Laparoscopic tool tracking, Optical pose tracker, Motion analysis, Motion analysis, Image-guided intervention.

\section{INTRODUCTION}

Image-guided interventions (IGI) have been greatly expanded by the advances in medical imaging and computing power over the past 20 years, driven by the surgical aim of progressively provide less invasive and harmful treatments. Tracking systems are an essential component of IGI systems for determining the spatial relationship between the surgical instruments, the anatomy, and the preoperative information. This is a useful tool for surgeons when the surgical instrument is outside the field of view, obscured by artifacts or occlusions, or when the instrument cannot be detected by the imaging system [1]. However, as of today, further efforts are needed in order to provide affordable and accurate tracking systems for laparoscopic instruments that can be used in a real interventional site.

On the other hand, minimally invasive surgery is a high demanding surgical approach concerning technical requirements for the surgeon, which must be trained in order to perform a safe surgical intervention. Traditional surgical education in minimally invasive surgery is commonly based on subjective criteria to quantify and evaluate surgical abilities. However, researchers, surgeons and associations are increasingly demanding the development of more objective training and assessment tools that can accredit surgeons as technically competent [2]. As has been reported in the literature, laparoscopic instrument motion analysis can be a suitable solution for developing automatic objective assessment tools for assessment of surgical technical skills

In a previous work, we proposed a tracking system in order to address these two concerns [3]. It applies a third generation optical pose tracker (MicronTracker ${ }^{\circledR} \mathrm{Hx} 60$; Claron Technology Inc., Toronto, CAN) to laparoscopic practice for both motion analysis of laparoscopic instruments for surgical assessment and image-guided applications.

This first version enabled tracking with real laparoscopic instruments while allowing users to grip and use the instruments in a natural way. Reported accuracy results showed stable but low positional accuracy to track the instrument tip. These results could be enough for objective assessment of skills based on instrument motion; however, accuracy is a crucial issue in order to use tracking systems in IGI.

The main objective of this study is to improve the accuracy of this laparoscopic instrument tracking system based on a third generation optical pose tracker. We hypothesize that this accuracy improvement could be achieved with a design enhancement of the artificial markers placed on the laparoscopic instrument and an improvement of the calibration process. To this end, a comparative study of positional accuracy between the previous design and the new approach is presented.

\section{MATERIALS AND METHODS}

\section{A. System Description}

Departing from the first version of the tracking system, a new support for three artificial markers (one at the front 
and two in both sides) was designed in order to track each laparoscopic instrument. This support was placed on the handle of each instrument to avoid not disturbing the natural use of the laparoscopic instruments. The material chosen for the support (ABS polymer) is lightweight (12 g) and tough.

In order to increase the system accuracy of the previous design [3], equation (1) was taken into account to estimate the error in computing the position of the instrument tip (provided by the manufacturer). This equation shows that the only two possible ways for error reduction are increasing the distance between markers placed on the support $(l)$ or decreasing the distance between the support and the instrument tip $(d)$. The latter option entails placing the markers on the instrument shaft or inserting a foreign body inside the patient or simulator. Consequently, it was decided to increase the separation between markers (Fig. 1a).

$$
\begin{gathered}
e_{\text {tip }} \approx e_{m}+1.5 \cdot e_{m} \cdot d / l \\
e_{m}=e_{\text {calibration }}+e_{\text {jitter }}=0.35 \mathrm{~mm}+0.14 \mathrm{~mm}=0.49 \mathrm{~mm}
\end{gathered}
$$

One of the challenges in computing the laparoscopic instrument tip is that it is difficult to determine the exact position of its centre point. The tip of each laparoscopic instrument is different and with irregular shape. To solve this problem an additional support for laparoscopic instruments was designed (Fig. 1b), which covers the distal part and provides us a fixed point of reference $\left(P^{\prime}\right)$ to be used during the calibration process.

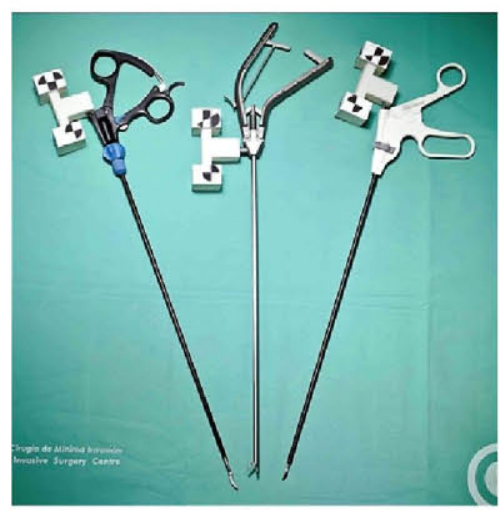

(a)

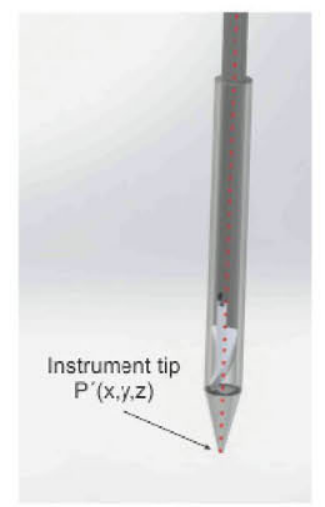

(b)
Fig. 1 (a) Set of laparoscopic instruments with the new marker supports. (b) Support for computing the instrument tip with regard to the central axis of the instrument shaft

For the calibration process the aforementioned support (Fig. lb) was used, as well as a calibration plate under the tip in order to compute the transformation matrices from the markers to the same point of reference $\left(P^{\prime}\right)$.

\section{B. Technical Evaluation}

An adaptation of the methodology defined by Hummel et al. [4] has been used to verify the positional accuracy and quantify the effects of noise of both tracking designs. A laparoscopic dissector (Richard Wolf $\mathrm{GmbH}$, Knittlingen, Germany) was used to perform all technical evaluation tests. The camera system was placed at $600 \mathrm{~mm}$ from the working area and its height was established at $340 \mathrm{~mm}$, which is approximately the same as the height of the markers on the instrument.

To technically validate the new system design and compare the accuracy results with the previous approach, two measurement platforms were developed. One platform for positional accuracy assessment was built with modified bricks and building plates of LEGO ${ }^{\circledR}$ (LEGO 6176 DUPLO Basic Bricks), which provides precise measures. The size of each basic piece used is $31.75 \mathrm{~mm}$ (Fig. 2a). To test inclination accuracy, a similar platform to the one proposed for the first version of the tracker was used [3]. The platform features 13 positions for the instrument at intervals of $7.5 \mathrm{de}-$ grees (Fig. 2b). Its configuration has been adapted to be used with the additional support for the instrument tip (Fig. lb), and therefore provide a stable position of the instrument tip throughout the evaluation test.

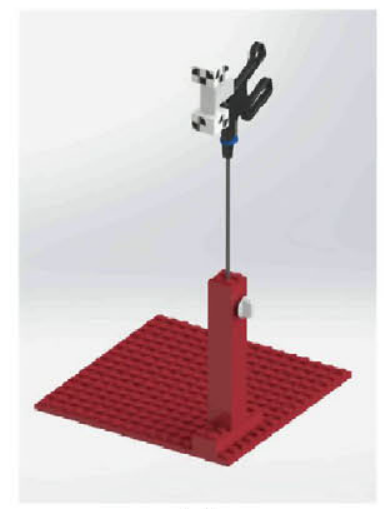

(a)

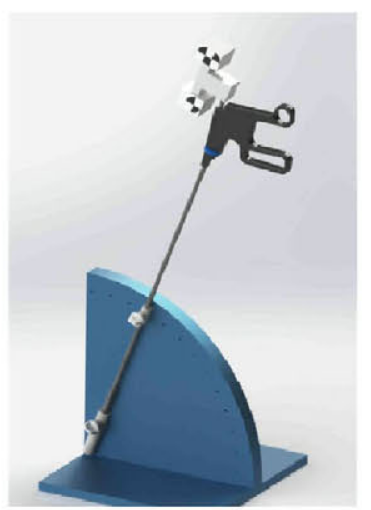

(b)
Fig. 2 Evaluation platforms. (a) Platform for the positional assessment. (b) Platform for the inclination assessment

For the evaluation test of positional accuracy the instrument tip was placed in 64 positions on the measurement platform distributed as a grid. For the inclination test the instrument was placed at each degree of inclination and with the tip fixed at the same origin. For both evaluation tests the position of the instrument tip was recorded during 10 seconds at each position. 
The fluctuation error at each position was computed by the root mean square error (RMSE). The accumulated distance error was computed by means of obtaining the distances from the first position of the measurement platform $P(i, 1)_{i=1,2, \ldots, 8}$ to all other column positions $P(i, j)_{j=1,2, \ldots, 8}$, and comparing them with the real distances. Relative position errors were computed by comparing the Euclidean distances reported by the tracking system to the known physical distances on the measurement platforms. Possible distances on the platform were computed with regard to multiples of a Displacement Unit (DU) of $31.75 \mathrm{~mm}$.

\section{RESULTS}

Figure 3 shows the graphs for the fluctuation error for both designs. The maximum fluctuation error for the new design $(0.536 \mathrm{~mm})$ is considerably lower than the previous approach $(2.991 \mathrm{~mm})$. In addition, for the new design this error is more stable throughout the working area than the previous one.
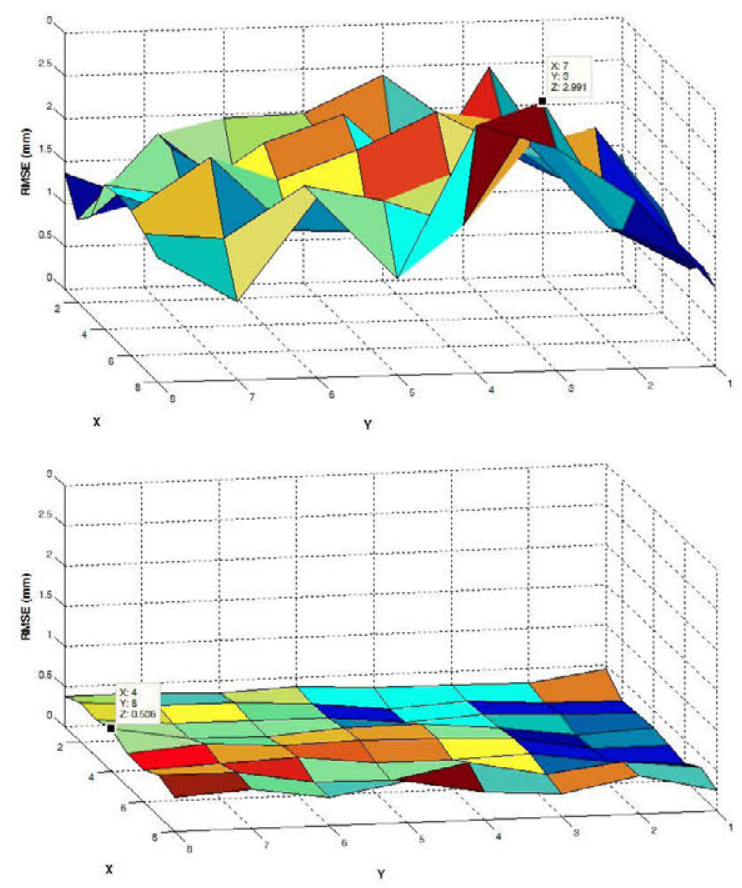

Fig. 3 Fluctuation error. (Top) Previous design. (Bottom) New design

As we expected, the accumulated distance error increases with the distance from the camera system for both designs (Fig. 4). However, the new design has a more linear distribution and its maximum error is lower $(8.205 \mathrm{~mm}$ for the previous design versus $5.448 \mathrm{~mm}$ for the new approach). Both designs present their maximum errors at the last row of the measurement platform.
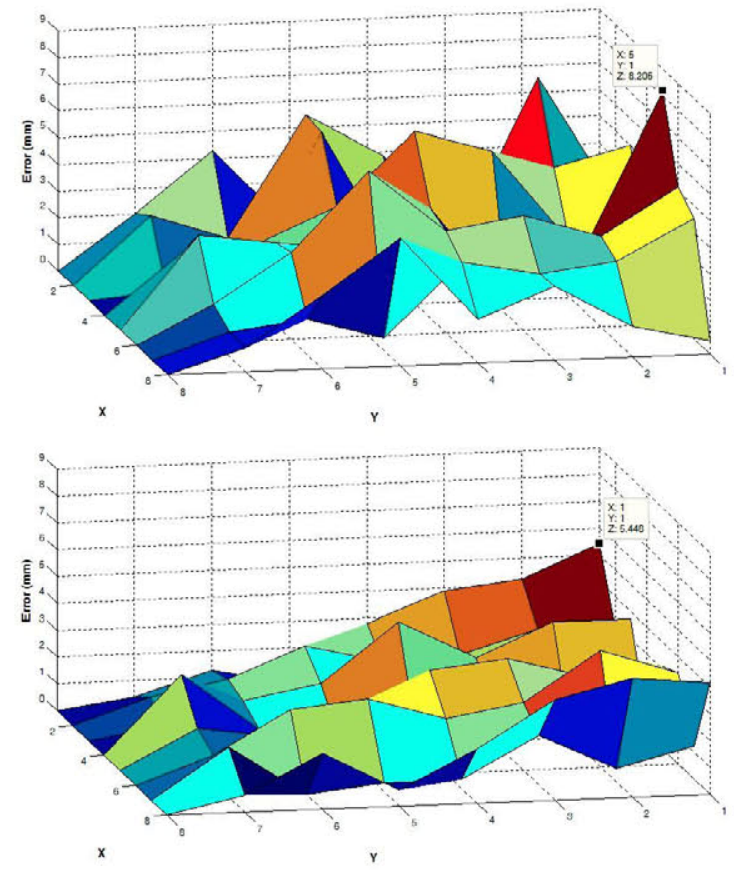

Fig. 4 Accumulated distance error. (Top) Previous design. (Bottom) New design

Table 1 Relative error (in mm). DU: Displacement Unit (31.75 mm). SD: Standard Deviation

\begin{tabular}{lcccc}
\hline \multirow{2}{*}{ Distance } & \multicolumn{2}{c}{ Previous design } & \multicolumn{2}{c}{ New design } \\
\cline { 2 - 5 } & Mean & Max. & Mean & Max. \\
\hline $1 * \mathrm{DU}$ & $2.149 \pm 1.713$ & 7.782 & $0.933 \pm 0.725$ & 1.778 \\
$2 * \mathrm{DU}$ & $2.377 \pm 1.767$ & 8.702 & $1.165 \pm 0.857$ & 2.198 \\
$3 * \mathrm{DU}$ & $2.372 \pm 1.879$ & 6.976 & $1.540 \pm 1.034$ & 2.873 \\
$4 * \mathrm{DU}$ & $2.500 \pm 1.648$ & 6.470 & $1.953 \pm 1.059$ & 3.362 \\
$5 * \mathrm{DU}$ & $2.467 \pm 1.802$ & 9.668 & $2.238 \pm 1.083$ & 3.797 \\
$6 * \mathrm{DU}$ & $3.140 \pm 1.671$ & 7.142 & $2.437 \pm 1.315$ & 4.851 \\
$7 * \mathrm{DU}$ & $3.373 \pm 2.057$ & 8.205 & $2.898 \pm 1.329$ & 4.935 \\
\hline
\end{tabular}

Table 1 shows the relative error rates. In general, this error is lower for the new design for all analyzed distances. This reduction is more noticeable at short distances (from $1 *$ DU to $4 *$ DU). Moreover, maximum errors at each evaluated distance are decreased.

The tilt range covered by both systems goes from 90 to 7.5 degrees. For the new design there is a reduction of both fluctuation error and accumulated distance error at each position. For both designs the maximum fluctuation error and accumulated distance error take place at the most horizontal position of the instrument $\left(7.5^{\circ}\right)$. 
Table 2 Inclination error (in mm). RMSE: Root mean square error. ADE: Accumulated distance error

\begin{tabular}{lcccc}
\hline \multirow{2}{*}{ Degree } & \multicolumn{2}{c}{ Previous design } & \multicolumn{2}{c}{ New design } \\
\cline { 2 - 5 } & RMSE & ADE & RMSE & ADE \\
\hline 90.0 & 0.568 & - & 0.266 & - \\
82.5 & 0.589 & 1.807 & 0.339 & 0.124 \\
75.0 & 0.688 & 1.136 & 0.269 & 0.245 \\
67.5 & 0.756 & 1.382 & 0.293 & 0.513 \\
60.0 & 0.640 & 1.328 & 0.354 & 0.935 \\
52.5 & 0.592 & 1.014 & 0.352 & 0.731 \\
45.0 & 0.692 & 0.651 & 0.416 & 0.923 \\
37.5 & 0.615 & 1.988 & 0.437 & 0.979 \\
30.0 & 0.431 & 1.907 & 0.399 & 1.067 \\
22.5 & 0.532 & 0.581 & 0.491 & 1.122 \\
15.0 & 0.737 & 1.294 & 0.477 & 1.292 \\
7.5 & 0.880 & 2.775 & 0.583 & 1.337 \\
\hline
\end{tabular}

\section{DISCUSSION}

This study presents the improved version of an original approach to laparoscopic instrument tracking based on a third dimensional optical pose tracker. This kind of tracking systems is fully passive and use light in the visible spectrum to identify targets. In general, they are more affordable than commercial IR-based systems, have no wires hanging from the instrument, and suffer no interference from metallic objects as in the case of electromagnetic tracking systems.

The new approach improves the accuracy results of a previous design [3], and therefore enables it to be used for motion analysis of laparoscopic instruments in training/ assessment and IGI applications. This system design does not interfere with the natural use of the instrument during surgery and nor increase its weight. It tries to address the need of tracking systems more robust and transparent for the user. Nevertheless, the use of video-based tracking systems has some challenges that need to be tackled. As reported by Maier-Hein et al. [5] these systems have some difficulties concerning poor lighting conditions and fast movements. Possible solutions to these limitations will be sought combined with computer vision techniques and pattern recognition techniques [6]. Moreover, to avoid line-ofsight obstructions, ergonomic studies are being carried out to determine optimal placement of the camera system in OR settings.

Despite these shortcomings, presented evaluation tests give proof of its accuracy and robustness, improving even the results from the first prototype presented [3]. Current works are focusing both on the analysis of the dynamic positional error of the system, as well as a complete validation study of the tracking system in a real scenario for MIS skills assessment.

\section{CONCLUSIONS}

The presented work offers a new tracking solution of laparoscopic instruments for objective evaluation of surgical technical skills and IGI. The system does not disturb the natural use of the surgical instruments. Both the design of the artificial markers on the instrument and the calibration process has been optimized as a means to improve the positional accuracy of the system with regard to a previous approach. Results have shown that this new design provides a general reduction both of positional and inclination error. The next step in order to comprehensively assess this system for clinical applications will be to analyze the dynamic positional error and its validation in a real scenario for training and assessment of MIS skills.

\section{ACKNOWLEDGMENT}

This study was supported in part by Gobierno de Extremadura, Consejería de Empleo, Empresa e Innovación, and the European Social Fund.

\section{REFERENCES}

1. K Cleary and TM Peters (2010) Image-guided interventions: technology review and clinical applications. Annu Rev Biomed Eng 12: 11942.

2. van Hove PD, Tuijthof GJM, Verdaasdonk EG et al. (2010) Objective assessment of technical surgical skills. Br J Surg 97(7): 972-987.

3. Sánchez-Margallo JA, Sánchez-Margallo FM, Pagador JB et al. (2013) Technical Evaluation of a Third Generation Optical Pose Tracker for Motion Analysis and Image-Guided Surgery. Lect Notes Comput Sci 7761:75-82.

4. Hummel JB, Bax MR, Figl ML et al. (2005) Design and application of an assessment protocol for electromagnetic. Med Phys 32(7): 2371-9.

5. Maier-Hein L, Franz A, Meinzer HP et al. (2008) Comparative assessment of optical tracking systems for soft tissue navigation with fiducial needles. Proc. SPIE. vol. 6918, San Jose, CA, USA, 2008, pp 69181Z-69181Z-9.

6. Sánchez-Margallo JA, Sánchez-Margallo FM, Pagador JB et al. (2011) Video-based assistance system for training in minimally invasive surgery. Minim Invasive Ther Allied Technol 20(4): 197-205.

\footnotetext{
Author: Juan Alberto Sánchez Margallo Institute: Jesús Usón Minimally Invasive Surgery Centre Street: Ctra N-521, km 41,8. 10071

City: Cáceres

Country: Spain

Email: jasanchez@ecmijesususon.com
} 